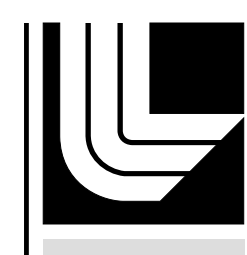

LAWRENCE LIVERMORE N A T IO N A L LABORATORY

\title{
GAMMA-RAY COMPTON LIGHT SOURCE DEVELOPMENT AT LLNL
}

F.V. Hartemann, S.G. Anderson, D.J. Gibson, C.A. Hagmann, M.S. Johnson, I. Jovanovic, M.J. Messerly, J.A. Pruet, M.Y. Shverdin, A.M. Tremaine, D.P. McNabb, C.W. Siders, C.P.J. Barty

August 22, 2007

IEEE/PPPS 2007

Albuquerque, NM, United States

June 17, 2007 through June 22, 2007 
This document was prepared as an account of work sponsored by an agency of the United States Government. Neither the United States Government nor the University of California nor any of their employees, makes any warranty, express or implied, or assumes any legal liability or responsibility for the accuracy, completeness, or usefulness of any information, apparatus, product, or process disclosed, or represents that its use would not infringe privately owned rights. Reference herein to any specific commercial product, process, or service by trade name, trademark, manufacturer, or otherwise, does not necessarily constitute or imply its endorsement, recommendation, or favoring by the United States Government or the University of California. The views and opinions of authors expressed herein do not necessarily state or reflect those of the United States Government or the University of California, and shall not be used for advertising or product endorsement purposes. 


\title{
GAMMA-RAY COMPTON LIGHT SOURCE DEVELOPMENT AT LLNL *
}

\author{
F.V. Hartemann ${ }^{\xi}$, S.G. Anderson, D.J. Gibson, C.A. Hagmann, M.S. Johnson, I. Jovanovic, \\ M.J. Messerly, J.A. Pruet, M.Y. Shverdin, A.M. Tremaine, D.P. McNabb C.W. Siders, and \\ C.P.J. Barty \\ Lawrence Livermore National Laboratory, Livermore, CA 94550 USA
}

\section{Abstract}

A new class of tunable, monochromatic $\gamma$-ray sources capable of operating at high peak and average brightness is currently being developed at LLNL for nuclear photoscience and applications. These novel systems are based on Compton scattering of laser photons by a high brightness relativistic electron beam produced by an $\mathrm{rf}$ photoinjector. A prototype, capable of producing $>10^{8} 0.7$ $\mathrm{MeV}$ photons in a single shot, with a fractional bandwidth of $1 \%$, and a repetition rate of $10 \mathrm{~Hz}$, is currently under construction at LLNL; this system will be used to perform nuclear resonance fluorescence experiments. A new symmetrized S-band rf gun, using a $\mathrm{Mg}$ photocathode, will produce up to $1 \mathrm{nC}$ of charge in an $8 \mathrm{ps}$ bunch, with a normalized emittance modeled at $0.8 \mathrm{~mm}$.mrad; electrons are subsequently accelerated up to $120 \mathrm{MeV}$ to interact with a $500 \mathrm{~mJ}, 10 \mathrm{ps}, 355 \mathrm{~nm}$ laser pulse and generate $\gamma$ rays. The laser front end is a fiber-based system, using corrugated-fiber Bragg gratings for stretching, and drives both the frequency-quadrupled photocathode illumination laser and the $\mathrm{Nd}$ :YAG interaction laser. Two new technologies are used in the laser: a hyper-Michelson temporal pulse stacker capable of producing 8 ps square UV pulses, and a hyper-dispersion compressor for the interaction laser. Other key technologies, basic scaling laws, and recent experimental results will also be presented, along with an overview of future research and development directions.

\section{I.INTRODUCTION}

Recent advances in high brightness rf gun and fiber laser technology have enabled the development of a new class of compact, tunable, narrow-bandwidth light sources capable of producing $\mathrm{MeV}$ photons with unprecedented brightness. Such new sources rely on Compton scattering of incident photons produced by a TW-class laser off a bright relativistic electron beam to generate Dopplerupshifted photons in a highly collimated beam. The main goal of this paper is to present a technical overview of socalled T-REX (Thomson-radiated extreme x-ray) sources and their key capabilities, and to provide an update on our progress.

\section{COMPTON SCATTERING}

Incident photons, with 4-wavenumber $k_{\mu}=\left(\omega_{0} / c, \mathbf{k}_{0}\right)$, can Compton scatter off electrons with initial 4-momentum $m_{0} c u_{\mu}$, to be Doppler-upshifted according to the Compton formula, which is derived from 4-momentum conservation: $\hbar k_{\mu}+m_{0} c u_{\mu}=\hbar q_{\mu}+m_{0} c v_{\mu}$, where $q_{\mu}$ is the 4-wavenuber of the scattered photon, and $m_{0} c v_{\mu}$ is the electron 4-momentum after the interaction. This leads to:

$$
\omega_{x}=\frac{\gamma \omega_{0}-\mathbf{u} \cdot c \mathbf{k}_{0}}{\gamma+\hbar_{C} k_{0}-\mathbf{n}_{x} \cdot\left(\mathbf{u}+\hbar_{C} \mathbf{k}_{0}\right)}
$$

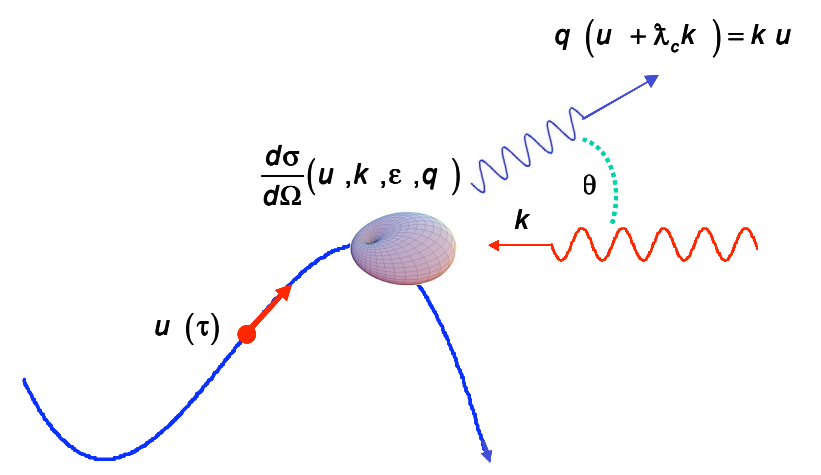

Figure 1 Compton scattering

Here, $\omega_{x}$ is the frequency of the scattered photon, and $\mathbf{n}_{x}$ is a unit vector in the direction of observation; $\lambda_{C}=\hbar / m_{0} c$ is the Compton wavelength of the electron.

The differential cross-section for this process is described by the Klein-Nishina formula [2]. Limiting the expression to spin-unpolarized electron beams; introducing the classical electron radius, $r_{0}$; the incident and scattered light-cone variables $\kappa_{0}=u_{\mu} k^{\mu}$ and $\kappa_{x}=v_{\mu} q^{\mu}$; and the incident and scattered 4-polarizations, $\varepsilon_{\mu}^{0}$ and $\varepsilon_{\mu}^{x}$, one obtains:

\footnotetext{
* This work was performed under the auspices of the U.S. Department of Energy by University of California, Lawrence Livermore National Laboratory under Contract W-7405-Eng-48.

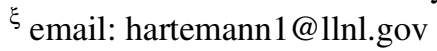




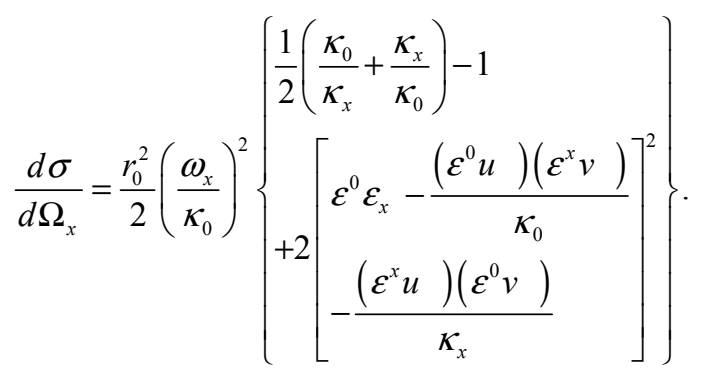

The brightness of Thomson scattering light sources was been studied theoretically [1] and computationally [3], and shown to scale favorably at high energy; this is due to the close correlation between the scattered light phase space and that of the incident electron beam: for a given normalized emittance, $\varepsilon_{n}$, the physical emittance of the electron beam scales as $\varepsilon_{n} / \gamma$; in turn, this implies that the scattered light is better collimated and has narrower bandwidth. For example, given the laser and electron beam parameters described in Table 1, the peak on-axis spectral brightness is shown in Fig. 2: the blue line corresponds to the analytical theory presented in Ref. [1], while the red squares are generated by a fully threedimensional code that has been extensively benchmarked against a series of detailed experiments at LLNL [3]; the maximum brightness is shown to scale as $\left(\gamma / \varepsilon_{n}\right)^{2}$.

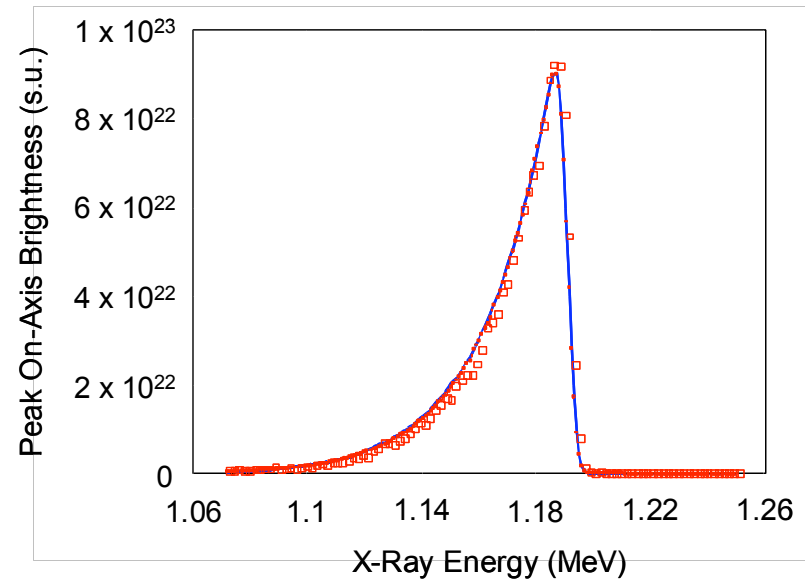

Figure 2 On-axis spectral brightness

\begin{tabular}{|l|l|l|}
\hline & Linac & Laser \\
\hline Particle energy & $250 \mathrm{MeV}$ & $\hbar \omega_{0}=1.24 \mathrm{eV}$ \\
\hline Particle \# & $q=N_{e} e=1 \mathrm{nC}$ & $W=1 \mathrm{~J}$ \\
\hline Pulse duration & $\Delta \tau / \sqrt{2}=100 \mathrm{fs}$ & $\Delta t=5 \mathrm{ps}$ \\
\hline Focal spot radius & $r_{b} / \sqrt{2}=10 \mu \mathrm{m}$ & $w_{0}=20 \mu \mathrm{m}$ \\
\hline Energy spread & $\Delta \gamma / \sqrt{2} \gamma=0.1 \%$ & $\Delta \omega=\sqrt{2} / \Delta t$ \\
\hline $\begin{array}{l}\text { Transverse phase } \\
\text { space }(\mathrm{rms})\end{array}$ & $\varepsilon_{n}=1 \mathrm{~mm} . \mathrm{mrad}$ & $\Delta k_{\perp} / k_{0} \ll 1$ \\
\hline
\end{tabular}

Table 1 Linac and laser parameters used for Fig. 2
For narrow bandwidth operation, the difference between the Compton and Thomson scattering formalisms becomes significant: this is shown in Fig. 3, where the relative frequency difference is plotted as a function of the beam energy for an incident wavelength of $1064 \mathrm{~nm}$; even for modest energies, recoil is sufficient to result in a frequency offset comparable to the scales of interest.

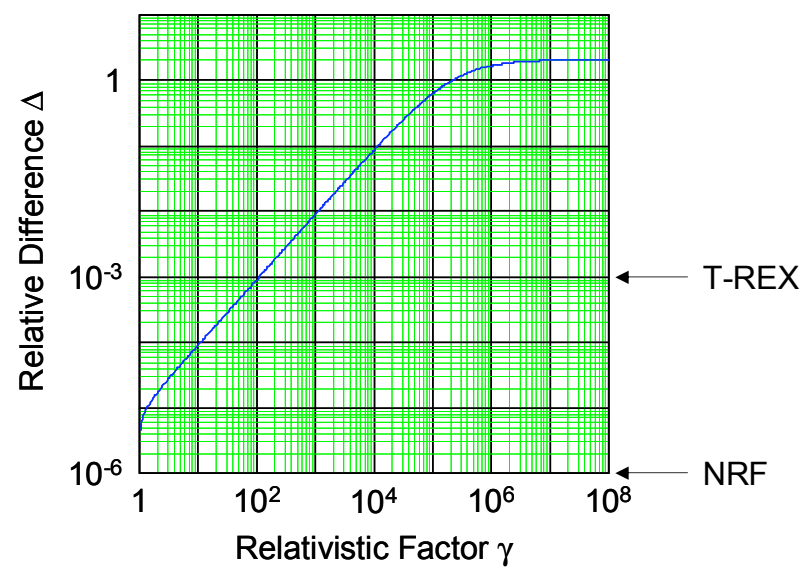

Figure 3 Relative recoil frequency offset as a function of the electron normalized energy, $\gamma$

\section{LLNL T-REX Source}

A prototype T-REX source is currently under construction at LLNL; it is designed to perform proof-ofprinciple nuclear resonance fluorescence (NRF) imaging experiments at photon energies up to $700 \mathrm{keV}$ [4]. The overall system architecture is shown in Fig. 4; key components include a high brightness S-band rf gun and associated fiber-based UV photocathode laser system, a $125 \mathrm{MeV}$ linac, and a Joule-class, $10 \mathrm{~Hz}$ drive laser, with frequency tripling down to $355 \mathrm{~nm}$. This source will produce $10^{9}$ photons/shot; the effective $\gamma$-ray beam divergence will be $0.5 \mathrm{mrad}$; and its fractional bandwidth will be $<1 \%$.

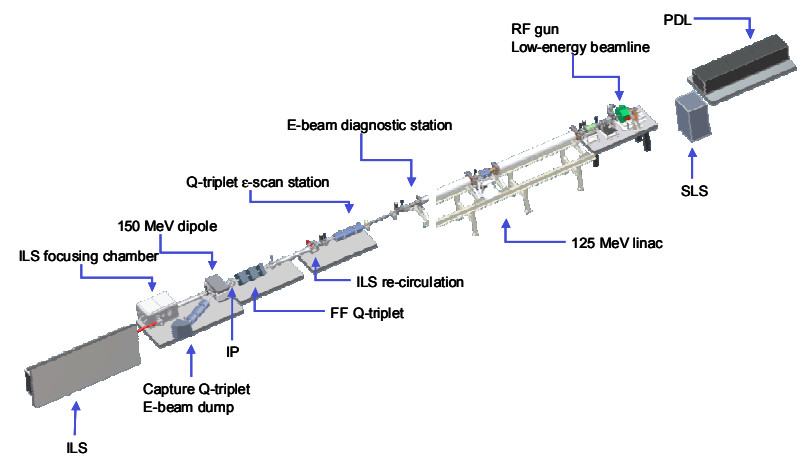

Figure 4 T-REX overall schematic

As indicated in the previous section, the x-ray phase space closely maps that of the electron beam; this implies that high charge, low emittance and low energy spread electron beams are critical to enable the development of 
high brightness Compton scattering light sources. Additional issues, such as the picosecond timing required between the electron and laser beams, also point in the direction of rf guns as the electron source of choice.

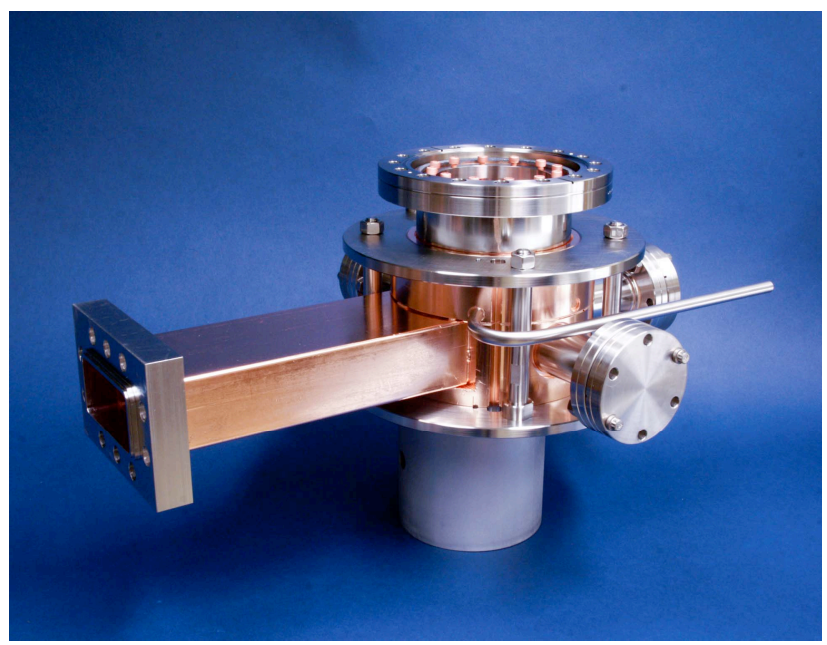

Figure 5 UCLA/LLNL S-band rf gun

One of the key characteristics of $\mathrm{rf}$ guns is the very high accelerating gradient under which the system operates: up to $120 \mathrm{MeV} / \mathrm{m}$ for good vacuum and processing conditions. This yields very bright photoelectron bunches that are naturally synchronized to the laser pulses used to illuminate the photocathode. However, to take full advantage of the unique properties of rf guns, one requires highly specific spatial and temporal laser pulse shapes at the photocathode, as shown in Fig. 6.

Given the stringent laser requirements for both the Thomson scattering drive laser and the rf gun photocathode illumination system, and the need for robustness, compactness, efficiency, and versatility, fiberbased systems are ideal candidates to meet the technical specifications of Compton light sources, especially in view of the fact that sputtered $\mathrm{Mg}$ can reduce the UV laser requirement down to a few tens or a few hundreds of $\mu \mathrm{J}$.
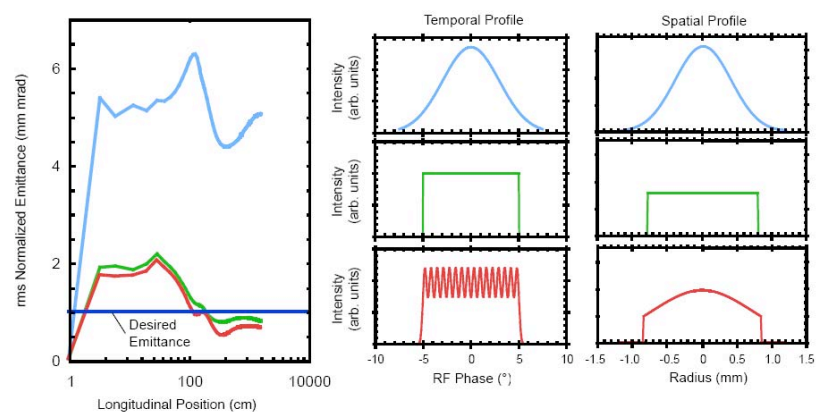

Figure 6 Influence of the UV pulse shape on emittance

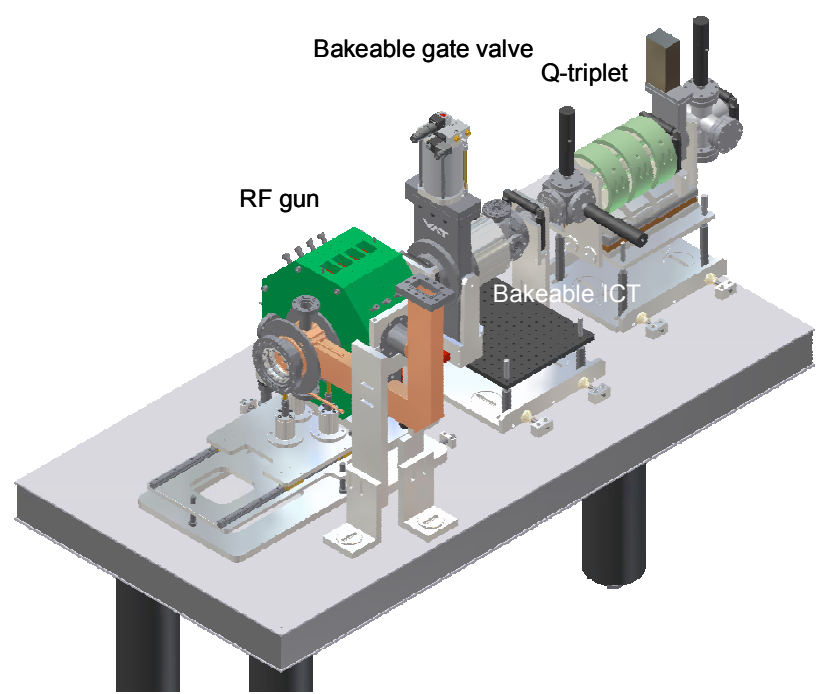

Figure 7 Low-energy beamline

Such novel lasers are highly stable, in part because of diode pumping; integrated optics, including fiber switches and chirped fiber Bragg gratings allow for the production and manipulation of ultrashort laser pulses, with energies up to $1 \mathrm{~mJ}$. Higher energies can be obtained by bulk amplification, while the fiber systems themselves are readily scaleable to multi-kW average powers. Moreover, using hyper-Michelson interferometers [5], it is possible to produce arbitrary and well-defined UV pulses (Fig. 8).

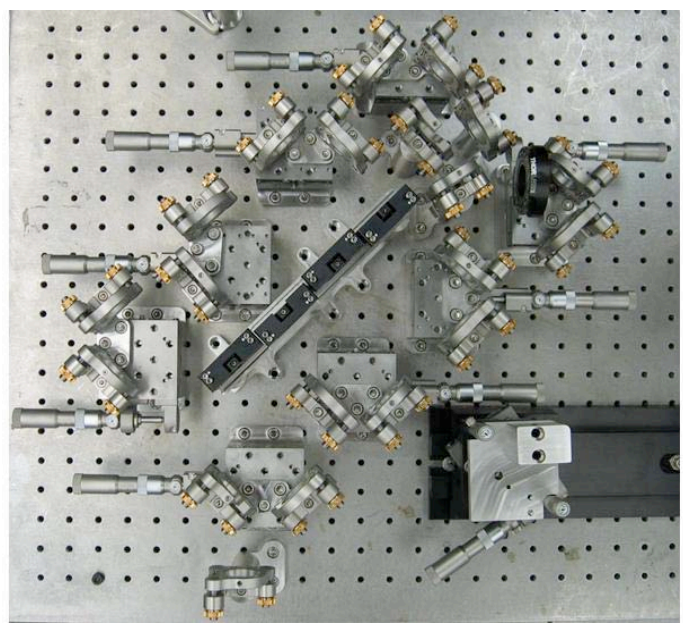

Figure 8 Hyper-Michelson

In Fig. 9, the IR pulse produced by the fiber-based system is analyzed using frequency-resolved optical gating (FROG), prior to frequency-quadrupling and injection into the hyper-Michelson temporal shaper. In addition, initial UV pulses have been produced, as shown in Fig. 10, with up to $5 \mu \mathrm{J}$ of energy; pulses at the $50 \mathrm{~mJ}$ level will produce $1 \mathrm{nC}$ of charge for the sputtered $\mathrm{Mg}$ photocathode, as previously measured in our lab (see Fig. 11). 


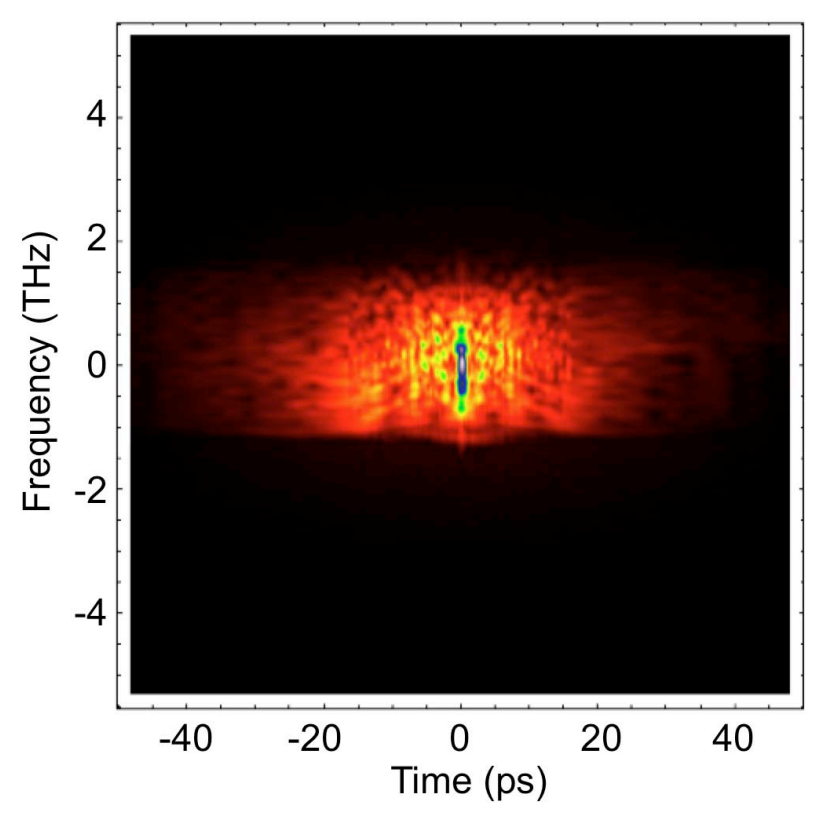

Figure 9 FROG of the compressed fiber-amplified pulse

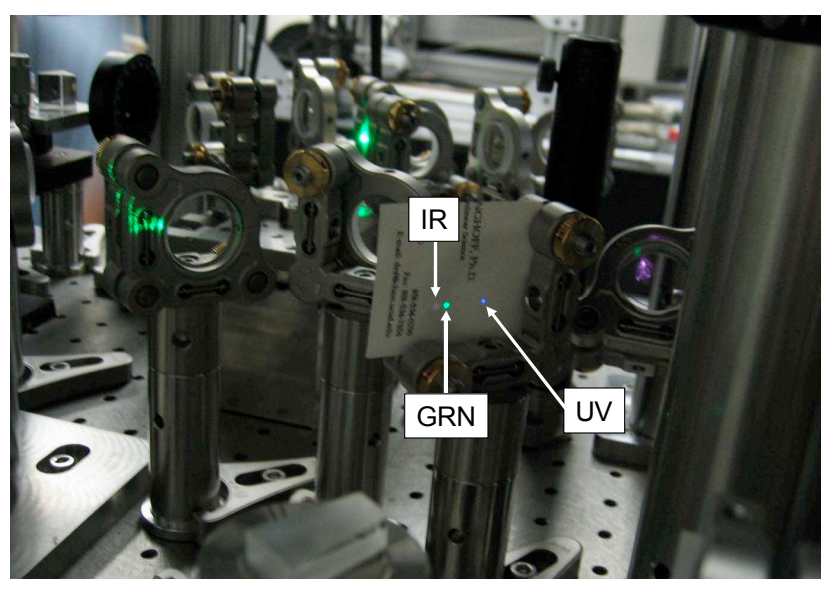

Figure 10 Initial UV pulses

Finally, a $25 \mathrm{~W}(2.5 \mathrm{~J}$ at $10 \mathrm{~Hz}) \mathrm{Nd}$ :glass amplification chain will be used to produce the drive pulses at the interaction region; this system is driven by a common fiber laser oscillator to ensure the required sub-ps synchronization.

The purpose of the interaction laser system (ILS) is to deliver Joule-class 355-nm pulses (the third harmonic of $\mathrm{Nd}$ :YAG) to the interaction region at $10 \mathrm{~Hz}$ repetition rate, synchronous with the photoinjector and linac. Importantly, the nominally picosecond duration achievable with Nd:YAG (limited by the available gain bandwidth) is much better suited for the generation of narrow-band $\gamma$-rays than the sub-picosecond pulses used previously in the PLEIADES project. The system consists of a seed source phase locked to the photocathode drive laser (PDL), a three-head Nd:YAG power amplifier, and a unique hyper-dispersion pulse compressor. The output from the pulse compressor is vacuum relay-imaged to the frequency tripling set-up.

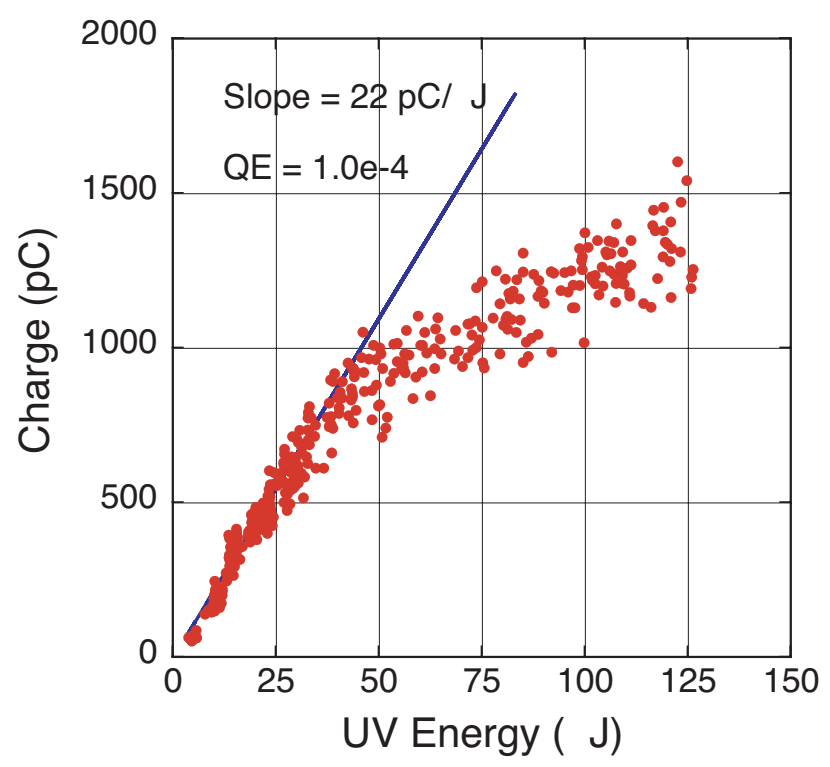

Figure 11 Sputtered $\mathrm{Mg}$ photocathode quantum efficiency measured at $80 \mathrm{MV} / \mathrm{m}$

The fiber-based seed laser system (SLS) is designed to deliver 1064-nm seed pulses temporally stretched to 6-ns duration in a double-passed chirped-fiber Bragg grating. These pulses are amplified to the $\mathrm{mJ}$ level in a multi-stage fiber amplifier, at which point they are available to be injected in the ILS power amplifier. Though amplification in Nd:YAG will naturally limit the bandwidth of the energetic output pulses, the ILS seed pulses are amplified with a $0.8 \mathrm{~nm}$ hard edge bandwidth in order to optimally fill the available gain spectrum.

The nominally 6-ns long 1064-nm pulses supplied by one arm of the SLS are quite similar to the seed pulses used in commercial Q-Switched Nd:YAG lasers. Such commercial lasers are typically limited to less than a handful of longitudinal modes as opposed to the widespectrum chirped pulses we will use. Since the bandwidth of the seed pulses is on the order of the available gain bandwidth of Nd:YAG, we use stock Nd:YAG amplifiers from a commercial vendor to boost the mJ-level output of the SLS fiber amplifiers to the Joule-level. Our design utilizes three commercial flashlamp-pumped $\mathrm{Nd}$ :YAG laser heads (Continuum Lasers): a dual $6 \mathrm{~mm}$ rod head and two $12 \mathrm{~mm}$ rod heads in a birefringence compensating configuration. In our design, the input incident pulse will traverse the $6 \mathrm{~mm}$ head four times and each of the $12 \mathrm{~mm}$ heads once. The multi-pass amplifier will boost up the input seed pulse from $500 \mu \mathrm{J}$ to an estimated output energy of $3 \mathrm{~J}$.

Chirped-pulse amplification in Nd:YAG with nanometer bandwidths is impractical using traditional two-grating pulse compressors due to the considerable spacing $(\sim 26 \mathrm{~m})$ required between the gratings. We have 
utilized a proprietary cascaded-grating "hyper-dispersion" architecture to provide the necessary dispersion $(\sim 3000$ $\mathrm{ps} / \mathrm{nm}$ ) in a compact meter-scale compressor.

Conceptually, our design uses four multi-layer dielectric (MLD) diffraction gratings in a double-pass configuration. The use of MLD gratings allows for high throughput efficiency in the compressor despite a total of eight grating reflections.

The beam is incident near the Littrow angle on all of the gratings. The first grating reflection angularly disperses the incident collimated beam. In traditional twograting compressors, the second grating collimates the dispersed beam. In our hyper-dispersion design, the second grating is oriented anti-parallel to the first grating, which additionally disperses the incident beam. The third and fourth gratings are arranged parallel, respectively, to the second and first gratings and collimate the spatially chirped beam.

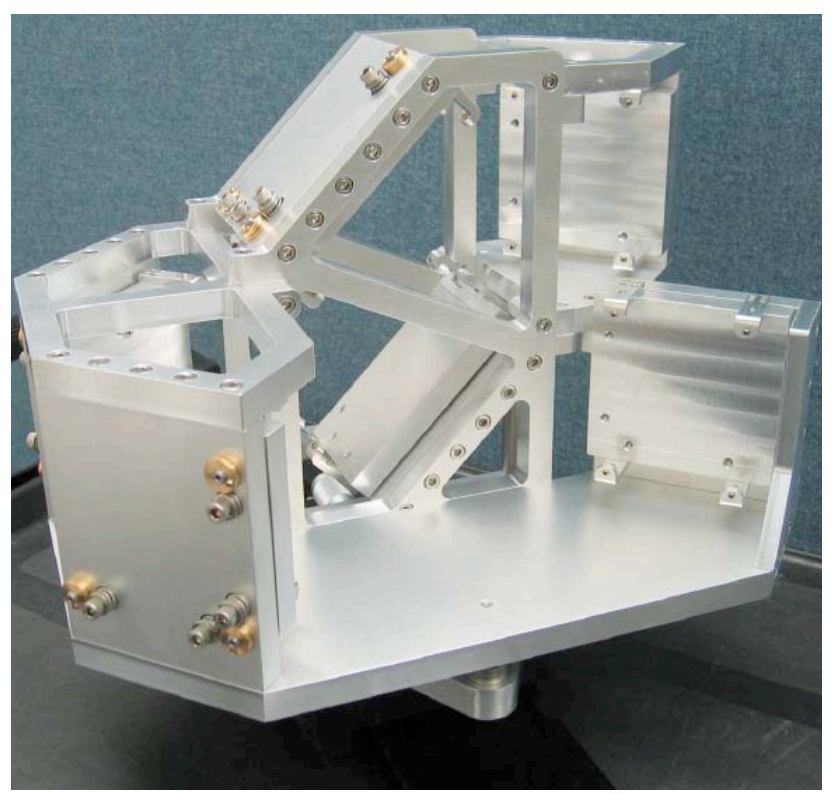

Figure 12 Hyper-dispersion compressor turning mirror assembly

Because multi-grating alignment can be complicated, we utilize appropriate folding of the beam path to reduce the total number of gratings used. In fact, a single grating design is possible with a very large area diffraction grating. We have chosen a hyper-dispersion compressor design that utilizes a total of two large-area $\left(40 \times 20 \mathrm{~cm}^{2}\right.$, 1740 grooves $/ \mathrm{mm}$ ) gratings. Each grating sees four beam reflections arranged in four rows. A key feature here is a 6 mirror assembly, which in addition to setting the height and the position of the beam also reflects the spatially chirped beam along the direction of propagation. After the six mirror assembly, the angularly dispersing beam reflects off the second and the first gratings and becomes collimated. Following the roof mirror, the beam retraces its path through the compressor.
The grating dimensions are determined by the total temporal chirp of the laser pulse and the damage fluence of the MLD gratings. To help minimize spatial modulation of the beam profile, the output of the multipass amplifier will be imaged to the final grating. A novel double relay telescope was developed to allow imaging through approximately $26 \mathrm{~m}$ of total beam path inside the compressor.

\section{References}

[1] F.V. Hartemann, et al., "High-energy scaling of Compton scattering light sources", Phys. Rev. STAB 8, 100702 (2005).

[2] G. Bhatt, et al., "Relativistic spin-dependent Compton scattering from electrons”, Phys. Rev. A28, 2195 (1983).

[3] W.J. Brown, et al., "Three-dimensional time and frequency-domain theory of femtosecond x-ray pulse generation through Thomson scattering", Phys. Rev. STAB 7, 060702; "Experimental characterization of an ultrafast Thomson scattering x-ray source", ibid 060703 (2004).

[4] J. Pruet, et al., "Detecting clandestine materials with nuclear resonance fluorescence", Jour. Appl. Phys. 99, 123102 (2006).

[5] C.W. Siders, et al., "Efficient high-energy pulse-train generation using a 2n-pulse Michelson interferometer", Appl. Opt. 37, 5302 (1998). 